\title{
A Randomized Controlled Trial of Puncturing and Bloodletting at Twelve Hand Jing Points to Treat Acute Carbon Monoxide Poisoning as Adjunct to First Aid Treatment: A Study Protocol
}

\author{
Ying Yue, ${ }^{1,2}$ Xingfang Pan, ${ }^{2}$ Sai Zhang, ${ }^{3}$ Jun Jin, ${ }^{4}$ Wei Wang, \\ Dongqiang Wang, ${ }^{5}$ Dexin Han, ${ }^{6}$ Guirong Wang, ${ }^{7}$ Qunliang Hu, ${ }^{3}$ \\ Jingqing Kang, ${ }^{3}$ Shasha Ding, ${ }^{2}$ Yi Yang, ${ }^{8}$ Huaien $\mathrm{Bu}^{,}{ }^{9}$ and $\mathrm{Yi} \mathrm{Guo}^{2}$ \\ ${ }^{1}$ Clinical Teaching and Training Department, Tianjin University of Traditional Chinese Medicine, Tianjin 300193, China \\ ${ }^{2}$ College of Acupuncture-Moxibustion and Tuina, Tianjin University of Traditional Chinese Medicine, Tianjin 300193, China \\ ${ }^{3}$ Neurosurgery and Neurology Hospital, Affiliated Hospital, Logistics University of the Chinese People's Armed Police Force, \\ Tianjin 300162, China \\ ${ }^{4}$ International Education College, Tianjin University of Traditional Chinese Medicine, Tianjin 300193, China \\ ${ }^{5}$ Department of Integration Traditional and Western Medicine, Tianjin First Central Hospital, Tianjin 300192, China \\ ${ }^{6}$ Emergency Department, Wuqing Affiliated Hospital, Tianjin University of Traditional Chinese Medicine, Tianjin 301700, China \\ ${ }^{7}$ Emergency Department, Tianjin Binhai New Area Dagang Hospital, Tianjin 300270, China \\ ${ }^{8}$ Research Centre for Standardization of Acupuncture-Moxibustion, School of Language and Culture, Tianjin University of \\ Traditional Chinese Medicine, Tianjin 300193, China \\ ${ }^{9}$ College of Traditional Chinese Medicine, Tianjin University of Traditional Chinese Medicine, Tianjin 300193, China
}

Correspondence should be addressed to Yi Guo; guoyi_168@163.com

Received 11 March 2015; Accepted 7 July 2015

Academic Editor: Shu-Ming Wang

Copyright (c) 2015 Ying Yue et al. This is an open access article distributed under the Creative Commons Attribution License, which permits unrestricted use, distribution, and reproduction in any medium, provided the original work is properly cited.

Background. Acute carbon monoxide poisoning (ACOP) is a significant cause of morbidity and mortality in many countries. Twelve Hand Jing Points (THJP) have been believed to be effective to treat all kinds of emergency calls in traditional Chinese medicine (TCM) for more than 3000 years. This randomized controlled trial (RCT) is designed to evaluate the effectiveness of THJP in curing acute carbon monoxide poisoning in first aid treatment. This paper reports the protocol of the trial. Methods/Design. This RCT is a multicenter, randomized, controlled study undergoing in China. The compliant patients are divided into the bloodletting group and standard of care group. With first aid treatments given to both of the groups, the bloodletting group is bleeding at THJP upon being hospitalized. Primary outcomes and secondary outcomes will be measured and compared between these two groups. Before treatment, immediately after treatment, and 30 minutes, 1 hour, and 4 hours after treatment, patients' basic vital signs and state of consciousness were observed. Before treatment and 1 and 4 hours after treatment, carboxyhemoglobin concentration in venous blood samples was detected. Discussion. The objective of this study is to provide convincing evidence to clarify the efficacy and safety of THJP for early treatment of acute carbon monoxide poisoning.

\section{Background}

Acute carbon monoxide poisoning (ACOP) is a significant cause of morbidity and mortality in many countries, the rate of the largest number of poisoning accidental poisoning of life and occupational poisoning $[1,2]$. It may account for more than half of the world fatal poisoning [3].

ACOP is rather common in winter of China. CO diffuses readily within the alveoli and has far greater affinity than oxygen for essential biological compounds such as hemoglobin 


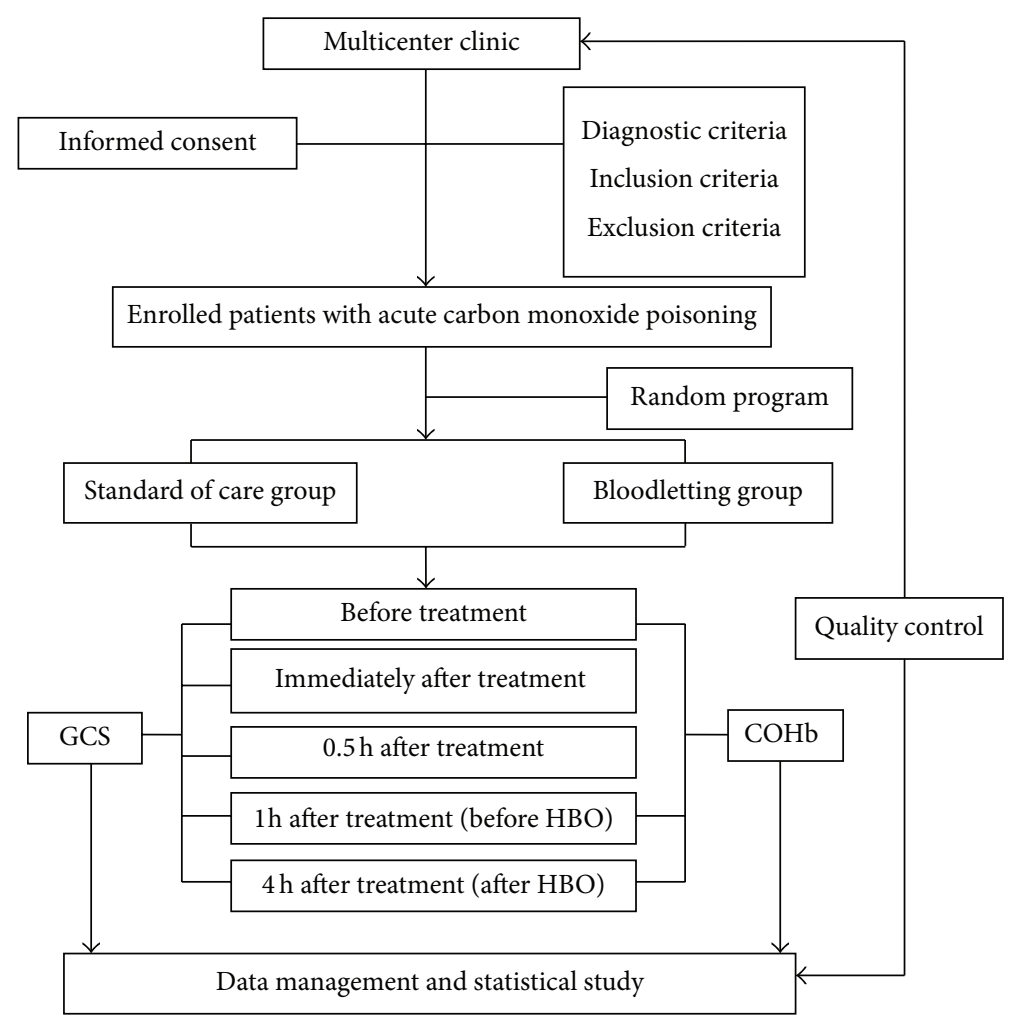

FIgURE 1: Trail flow chart.

and cellular oxidative enzymes. Thus, $\mathrm{CO}$ poisoning causes cellular hypoxia. However, the mechanisms of its complications remain unclear [4-6].

Although hyperbaric oxygen therapy (HBO) produces a larger reduction in carboxyhemoglobin half-life, its benefit is that risk ratio is still debated [7]. From the current research on acute carbon monoxide poisoning, researches on first aid measures right after the onset are few, but they can effectively solve hypoxia and protect the brain tissue [5].

Twelve Hand Jing Points are of the Chinese traditional medical first aid measures. They have been believed to treat all kinds of emergency for more than 3000 years. Previous studies have shown that THJP may be effective in the first aid treatment of acute carbon monoxide poisoning but have not provided conclusive evidence of THJP bloodletting for acute carbon monoxide poisoning [8]. Previous animal studies indicated that compared with the control group, the indicator of carboxyhemoglobin concentration and awaking time both significantly decreased with the mice receiving the THJP treatment with acute carbon monoxide poisoning $[9,10]$. For this study, the objective is to clarify the efficacy and safety of THJP in first aid treatment of acute carbon monoxide poisoning.

\section{Methods and Design}

2.1. Design. This is a multicenter randomized controlled trial, enrolling both men and women with acute carbon monoxide poisoning in China. This study will be conducted at Neurosurgery and Neurology Hospital, Affiliated Hospital, Logistics University of the Chinese People's Armed Police Force; Wuqing Affiliated Hospital, Tianjin University of Traditional Chinese Medicine, and Tianjin Binhai New Area Dagang Hospital. The patient or the patient's family should write the informed consent for the study. The compliant patients according to visit order, by the method of central random [11], are divided into the bloodletting group and standard of care group. A designated researcher will provide patient groups, and acupuncturists do not know patient groups before treatment. The acupuncturists only know the group assignment prior to the treatment (see Figure 1).

This trial is financed by the National Natural Science Foundation of China (number 81303023). The research protocol has been approved by the Clinical Trial Ethics Committee of Affiliated Hospital of Logistics University of Chinese People's Armed Police Forces (trial registration number: ChiCTR-IOR-15005797).

\subsection{Patients}

2.2.1. Study Population. Our study will include patients with acute carbon monoxide poisoning. To ensure the precision of results of this trial, patients who meet the following eligible criteria will be included in this study. 


\subsubsection{Inclusion Criteria}

(1) Meeting the diagnostic criteria of GBZ23-2002 in acute carbon monoxide poisoning.

(2) The course of disease is within 6 hours.

(3) After the poisoning patients with disturbance of consciousness.

\subsubsection{Exclusion Criteria}

(1) Patients with delayed encephalopathy after acute carbon monoxide poisoning, cerebral hernia.

(2) Liver and kidney function in previous severe abnormality.

(3) Pregnant women, diabetes, and mental patients.

(4) The blood coagulation dysfunction.

(5) Doctors belief that there are some cases not suitable for inclusion.

(6) Being not followed up.

\subsection{Intervention}

2.3.1. Standard of Care Group. The standard of care group will receive general first aid treatment including hyperbaric oxygen therapy (HBO) about 45-60 minutes, citicoline $1 \mathrm{~g}$ intravenously once daily, and naloxone $0.4 \mathrm{mg}$ intravenously every 12 hours.

2.3.2. Bloodletting Group. The bloodletting group will receive general first aid treatment, the same as control group. Meanwhile, the bloodletting group will be treated with THJP on admission immediately before hyperbaric oxygen therapy. The order of puncture and bloodletting should be set. In this study, we decide to puncture and bloodlet the left hand first and then the right hand in the order of Shaoshang (LU11), Shangyang (LI1), Zhongchong (PC9), Guan Chong (TE1), Shaochong (HT9), and Shaoze (S11). And the amount of bloodletting at each point is one drop. At the same time, we choose a unified model needle. The location of acupoints is as follows [12].

Shaoshang (LU11). It is on the thumb, radial to the distal phalanx, $0.1 \mathrm{~F}$-cun proximal-lateral to the radial corner of the thumb nail, at the intersection of the vertical line of the radial border and the horizontal line of the base of the thumb nail.

Shangyang (LI1). It is on the index finger, radial to the distal phalanx, $0.1 \mathrm{~F}$-cun proximal-lateral to the radial corner of the index fingernail, at the intersection of the vertical line of the radial border of the fingernail and the horizontal line of the base of the index fingernail.

Zhongchong (PC9). It is on the middle finger, at the centre of the tip of the middle finger, $0.1 \mathrm{~F}$-cun proximal-lateral to the radial corner of the thumbnail, at the intersection of the vertical line of the radial side of the nail and the horizontal line of the base of the finger nail.

Guan Chong (TE1). It is on the ring finger, ulnar to the distal phalanx, 0.1 F-cun proximal to the ulnar corner of the fingernail, at the intersection of the vertical line of the finger side of the nail and the horizontal line of the base of the finger nail.

Shaochong (HT9). It is on the little finger, radial to the distal phalanx, $0.1 \mathrm{~F}$-cun proximal-lateral to the radial corner of the little finger nail, at the intersection of the vertical line of the radial border of the nail and the horizontal line of the base of the little finger nail.

Shaoze (S11). It is on the little finger, ulnar to the distal phalanx, $0.1 \mathrm{~F}$-cun proximal-medial to the ulnar corner of the little finger nail, at the intersection of the vertical line of ulnar border of the nail and the horizontal line of the base of the little finger nail.

2.4. Outcome Measures. In this study, there are two primary outcomes and four secondary outcomes. These are presented in Table 1. Any adverse event resulting from THJP or adverse drug reaction will be recorded. Safety evaluation for THJP will be based on events which include hematoma, syncope, local infection, and any feelings of discomfort. Any adverse event resulting from THJP or adverse drug reaction to THJP will be recorded.

2.4.1. Primary Outcomes. Patients' states of consciousness (in Glasgow consciousness disorder table of GCS score) are observed at the following time points (before treatment, immediately after treatment, and 30 minutes, 1 hour, and 4 hours after treatment) in two groups. And patients' carboxyhemoglobin $(\mathrm{COHb})$ concentration of venous blood samples is detected at the following time points (before treatment and 1 hour and 4 hours after treatment) in two groups.

2.4.2. Secondary Outcomes. Patients' basic vital signs including temperature $(T)$, pulse $(P)$, respiration $(R)$, and blood pressure (BP) are observed at the following time points (before treatment, immediately after treatment, and $30 \mathrm{~min}$ utes, 1 hour, and 4 hours after treatment) in two groups.

2.5. Sample Size and Statistical Analysis. Sample size will be based on the primary outcome. According to our pilot trial and review of the literature [8], the average defecation rate of patient with $\mathrm{COHb}$ was $22.40 \%$ with a standard deviation of $3.65 \%$ and the average defecation rate of patient with $\mathrm{COHb}$ was $17.78 \%$ with a standard deviation of $5.68 \%$. To detect $\mathrm{COHb} \%$ difference, we need a sample size of 30 in each group $(\alpha=0.05, \beta=0.10)$ [13]. To allow 5\% for loss to follow-up, our proposed trial requires 62 patients (31 for each group).

Statistical analysis of all data will be done by Department of Public Health Statistics, which is blinded to this trial, using SPSS 19.0 software packages to analyze the data. All data are provided as mean \pm standard deviation. The level 
TABLE 1: Trial processes chart.

\begin{tabular}{|c|c|c|c|c|c|}
\hline \multirow{2}{*}{ Times } & \multicolumn{2}{|c|}{ Baseline } & \multicolumn{3}{|c|}{ Treatment } \\
\hline & -1 & 0 & $0.5 \mathrm{~h}$ & $1 \mathrm{~h}$ & $4 \mathrm{~h}$ \\
\hline \multicolumn{6}{|l|}{ Patients } \\
\hline Medical history & $\sqrt{ }$ & & & & \\
\hline Physical examination & $\sqrt{ }$ & $\sqrt{ }$ & $\sqrt{ }$ & $\sqrt{ }$ & $\sqrt{ }$ \\
\hline Laboratory examination & $\sqrt{ }$ & & & $\sqrt{ }$ & $\sqrt{ }$ \\
\hline Informed consent & $\sqrt{ }$ & & & & \\
\hline Randomization & $\sqrt{ }$ & & & & \\
\hline \multicolumn{6}{|l|}{ Intervention } \\
\hline Standard of care group & \multicolumn{5}{|c|}{ General first aid treatment } \\
\hline Bloodletting group & \multicolumn{5}{|c|}{ General first aid treatment + THJP } \\
\hline \multicolumn{6}{|l|}{ Outcome } \\
\hline \multicolumn{6}{|l|}{ primary outcomes } \\
\hline GCS & $\sqrt{ }$ & $\sqrt{ }$ & $\sqrt{ }$ & $\sqrt{ }$ & $\sqrt{ }$ \\
\hline $\mathrm{COHb}$ & $\sqrt{ }$ & & & $\sqrt{ }$ & $\sqrt{ }$ \\
\hline \multicolumn{6}{|l|}{ Secondary outcomes } \\
\hline $\mathrm{T}$ & $\sqrt{ }$ & $\sqrt{ }$ & $\sqrt{ }$ & $\sqrt{ }$ & $\sqrt{ }$ \\
\hline $\mathrm{P}$ & $\sqrt{ }$ & $\sqrt{ }$ & $\sqrt{ }$ & $\sqrt{ }$ & $\sqrt{ }$ \\
\hline $\mathrm{R}$ & $\sqrt{ }$ & $\sqrt{ }$ & $\sqrt{ }$ & $\sqrt{ }$ & $\sqrt{ }$ \\
\hline $\mathrm{BP}$ & $\sqrt{ }$ & $\sqrt{ }$ & $\sqrt{ }$ & $\sqrt{ }$ & $\sqrt{ }$ \\
\hline \multicolumn{6}{|l|}{ Trial evaluation } \\
\hline Safety of hand pricking blood therapy & $\sqrt{ }$ & $\sqrt{ }$ & $\sqrt{ }$ & $\sqrt{ }$ & $\sqrt{ }$ \\
\hline Adverse event & $\sqrt{ }$ & $\sqrt{ }$ & $\sqrt{ }$ & $\sqrt{ }$ & $\sqrt{ }$ \\
\hline Reasons of drop-outs or withdrawals & & & & & $\sqrt{ }$ \\
\hline Patient's compliance & & & & & $\sqrt{ }$ \\
\hline
\end{tabular}

of significance was set a $P$ value less than 0.05 . All of the analyses will be based on both an intention-to-treat analysis and a treated-per-protocol analysis. The intention-to-treat population was defined as the patients who are randomized and received at least one treatment session. The per-protocol population was defined as the patients who have no major protocol violations in the set. Both results of analysis will be compared to check whether the results are consistent.

\section{Discussion}

According to TCM theory, the body is regarded as a whole. It is composed of internal and external organs. These organs connect with each other by special ways called meridians [14]. Twelve Hand Jing Points are located at the end of fingers like the source of spring. Jing points are compared to the underground spring and the pulses here are so small.

Well or Jing is like underground water. It is described that the pulse is so small. The body of each of the twelve meridians has a well hole; they are also called Jing Points. Puncturing and bloodletting at Twelve Hand Jing Points are traditional Chinese medicine first aid measures used in various types of aid for more than 3000 thousand years in history. Some studies have indicated that acupuncture can play a therapeutic role in awaking consciousness disorders. From the clinical trials, we can see the potential for cerebral protection $[8,15-18]$. However, this deduction is insufficient and may lead to bias because of the poor quality which is without a standardized acupuncture protocol. Therefore, we designed a multicenter randomized controlled protocol and expected to provide convincing evidence to clarify the efficacy of THJP for first aid treatment of acute carbon monoxide. Acupuncture is a nontoxic, economical intervention with minimal adverse effects [19], which has been shown to remain effective even for a few months after the therapy [20]. One of the limitations in this trial design is the nonblinding of the patients and acupuncturists. Due to the characteristics of THJP, it is impossible to blind the patients and acupuncturists. However, we develop a rigorous methodology in other aspects of the study.

\section{Trial Status}

Twenty participants have been recruited.

\section{Disclosure}

Ying Yue and Xingfang Pan are joint first authors. 


\section{Conflict of Interests}

The authors declare that they have no conflict of interests.

\section{Authors' Contribution}

Ying Yue, Xingfang Pan, and Yi Guo conceived the design of the trial and Yi Guo revised it. Ying Yue and Huaien $\mathrm{Bu}$ participated in the design of the statistical analysis. Sai Zhang, Dexin Han, Guirong Wang, Qunliang Hu, Jingqing Kang, and Shasha Ding helped in the recruitment of patients. Jun Jin, Wei Wang, Yi Yang, and Dongqiang Wang helped in drafting the paper and quality control. All authors contributed to the final draft of the paper and decided to submit it for publication. All authors read and approved the final paper.

\section{Acknowledgment}

This trial is financed by the National Natural Science Foundation of China (no. 81303023).

\section{References}

[1] R. A. M. Meyers and S. R. Thom, "Carbon monoxide and cyanide poisoning," in Hyperbaric Medicine Practice, E. P. Kindwall, Ed., pp. 343-372, Best Publishing Company, 1995.

[2] J. A. Raub, M. Mathieu-Nolf, N. B. Hampson, and S. R. Thom, "Carbon monoxide poisoning-a public health perspective," Toxicology, vol. 145, no. 1, pp. 1-14, 2000.

[3] K. R. Hardy and S. R. Thom, "Pathophysiology and treatment of carbon monoxide poisoning," Clinical Toxicology, vol. 32, no. 6, pp. 613-629, 1994.

[4] L. K. Weaver, "Carbon monoxide poisoning," Critical Care Clinics, vol. 15, no. 2, pp. 297-317, 1999.

[5] K. Lindell and M. D. Weaver, "Carbon monoxide poisoning," The New England Journal of Medicine, vol. 360, pp. 1217-1225, 2009.

[6] C. D. Scheinkestel, M. Bailey, P. S. Myles et al., "Hyperbaric or normobaric oxygen for acute carbon monoxide poisoning: a randomised controlled clinical trial," Medical Journal of Australia, vol. 170, no. 5, pp. 203-210, 1999.

[7] D. Annane, K. Chadda, P. Gajdos, M.-C. Jars-Guincestre, S. Chevret, and J.-C. Raphael, "Hyperbaric oxygen therapy for acute domestic carbon monoxide poisoning: two randomized controlled trials," Intensive Care Medicine, vol. 37, no. 3, pp. 486492, 2011.

[8] J. Jin, S. Zhang, H. Li et al., "Effect of twelve well points of the hand pricking blood therapy on acute carbon monoxide poisoning state of consciousness in patients with disorders of consciousness," Journal of Emergency in Traditional Chinese Medicine, vol. 21, no. 2, pp. 175-176, 2012.

[9] J. Jin, Y. Yue, C. Qu et al., "Effects and mechanisms of stabbing collaterals bloodletting of the hand twelve well points on awaking in coma mice after carbon monoxide poisoning," Tianjin Journal of Traditional Chinese Medicine, vol. 29, no. 1, pp. 62-64, 2012.

[10] C. Qu, J. Jin, Y. Yue et al., "Effect of stabbing collaterals bloodletting of the hand twelve well points on the content of NE and cytochrome oxidase activity in mice after carbon monoxide poisoning," Journal of Tianjin University of Traditional Chinese Medicine, vol. 31, no. 1, pp. 27-30, 2012.
[11] Y. Cui, H. Bu, H. Wang, and S. Liao, "MACT: a manageable minimization allocation system," Computational and Mathematical Methods in Medicine, vol. 2014, Article ID 645064, 8 pages, 2014.

[12] WHO Standard Acupuncture Point Locations in the Western Pacific Region, WPRO, Manila, Philippines, 2009.

[13] J. Xu, L. Wang, and J. Xia, "Sample size estimation and statistical inference in a three-arm non-inferiority clinical trial including a placebo and an active control," Chinese Journal of Health Statistics, vol. 26, no. 3, pp. 255-257, 2009.

[14] C. H. Zheng, M. M. Zhang, W. Wang, and G. Y. Huang, "A randomized controlled trial of acupuncture to treat functional constipation: design and protocol," BMC Complementary and Alternative Medicine, vol. 14, article 423, 2014.

[15] D. Jing and G. Yi, "Effects of pricking blood at twelve Jing points of hand on state of consciousness in the patient of early stroke," Chinese Acupuncture \& Moxibustion, vol. 24, no. 10, pp. 673676, 2004.

[16] j. Dou and J. Jiang, "Progress in research on mechanism of early in thetreatment of ischemic stroke in the blood bleeding," Guiding Journal of Traditional Chinese Medicine and Pharmacy, vol. 20, no. 6, pp. 94-96, 2014.

[17] M. Zhang, Q.-L. Hu, S.-X. Cheng, Y. Tu, and S. Zhang, "Comparative study on the brain protection in patients of traumatic cerebral infarction treated with bloodletting at Jing-well points and semen coicis," Chinese Acupuncture \& Moxibustion, vol. 33, no. 9, pp. 779-783, 2013.

[18] Y. Guo, X. Wang, T. Xu et al., "Clinical observation of the influence of puncture and bloodletting at twelve Hand Jing Point on consciousness and heart rate in patients with wind-stroke," Tianjin Journal of Traditional Chinese Medicine, vol. 20, no. 2, pp. 37-38, 2003.

[19] WHO Library, Cataloguing-in-Publication Data: Acupuncture: Review and Analysis of Reports on Controlled Clinical Trials, WHO Library, 2002.

[20] C. P. O. Carlsson and B. H. Sjölund, "Acupuncture for chronic low back pain: a randomized placebo-controlled study with long-term follow-up," Clinical Journal of Pain, vol. 17, no. 4, pp. 296-305, 2001. 


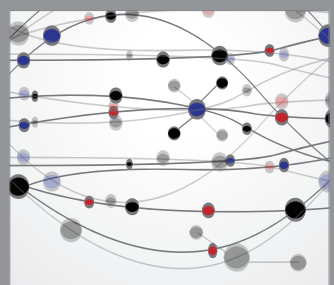

The Scientific World Journal
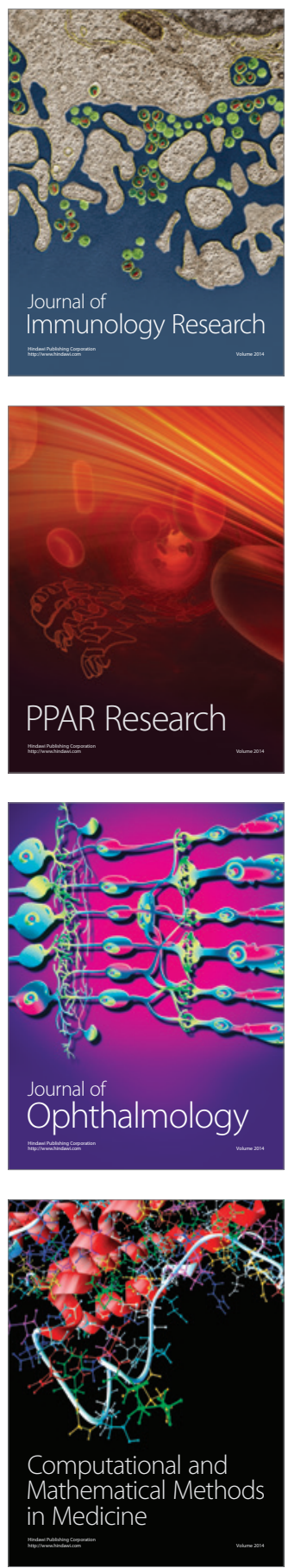

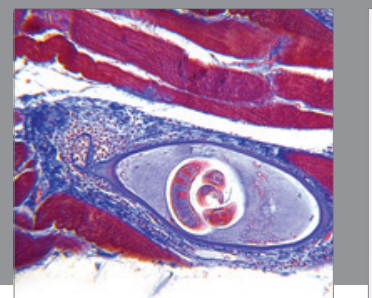

Gastroenterology

Research and Practice
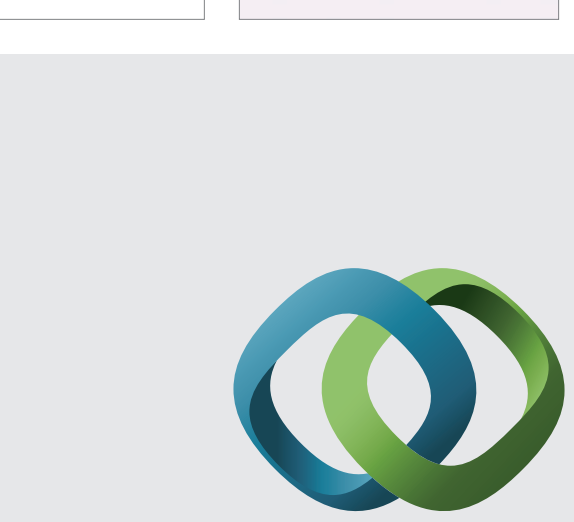

\section{Hindawi}

Submit your manuscripts at

http://www.hindawi.com
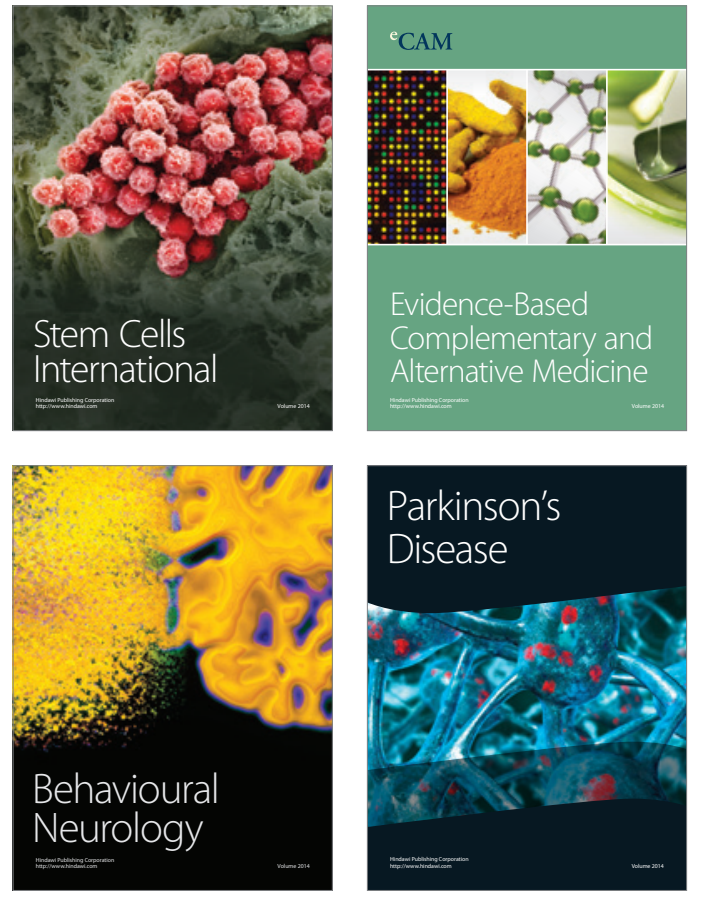
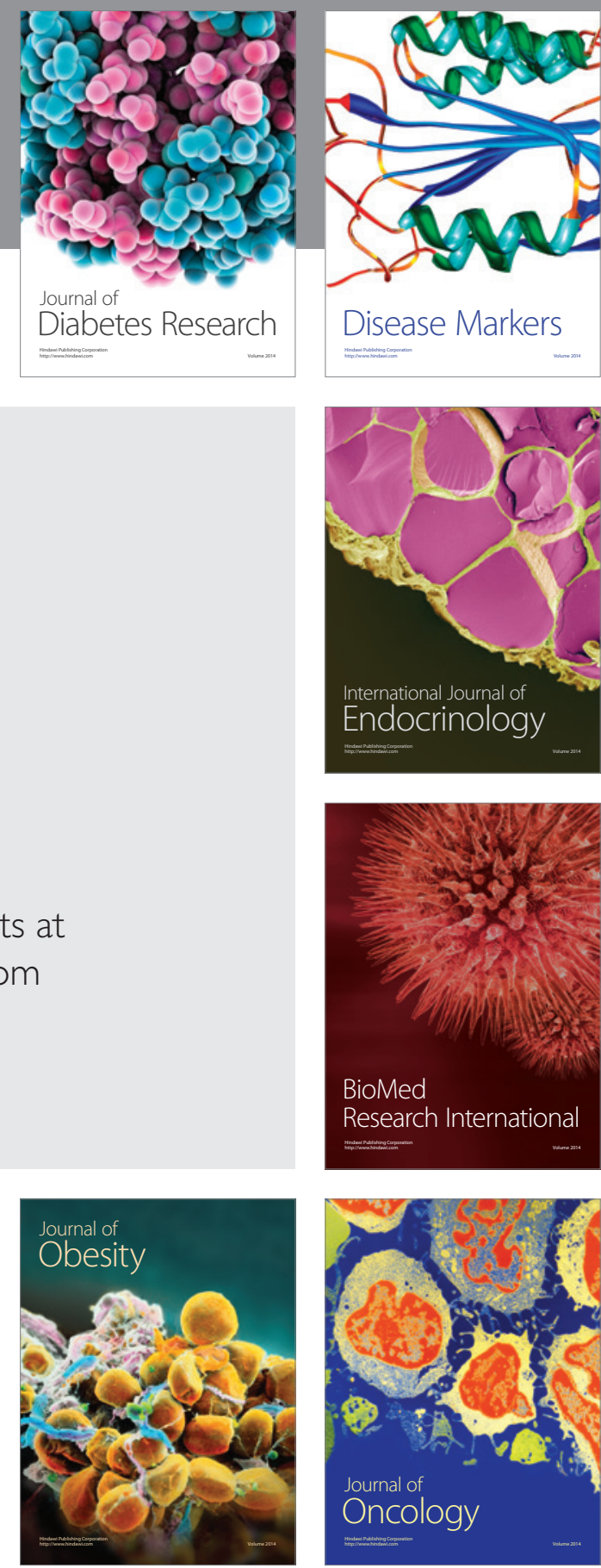

Disease Markers
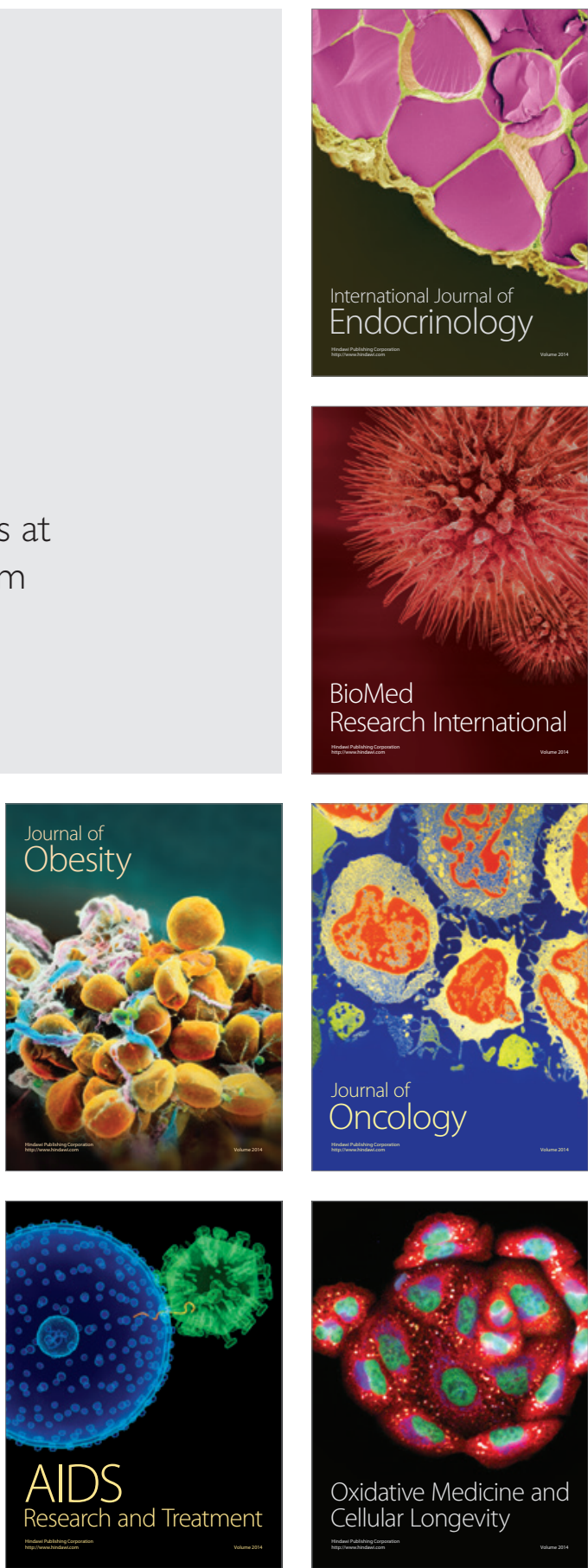